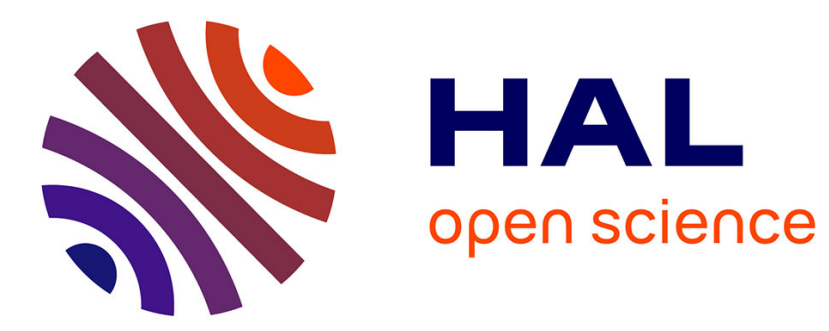

\title{
Le Sénat républicain représentant des collectivités territoriales
}

\author{
Tiphaine Le Yoncourt
}

\section{To cite this version:}

Tiphaine Le Yoncourt. Le Sénat républicain représentant des collectivités territoriales. Pouvoirs Revue française d'études constitutionnelles et politiques, 2016, Le Sénat, pour quoi faire?, 159, pp.2740. halshs-01426023

\section{HAL Id: halshs-01426023 https://shs.hal.science/halshs-01426023}

Submitted on 4 Jul 2019

HAL is a multi-disciplinary open access archive for the deposit and dissemination of scientific research documents, whether they are published or not. The documents may come from teaching and research institutions in France or abroad, or from public or private research centers.
L'archive ouverte pluridisciplinaire HAL, est destinée au dépôt et à la diffusion de documents scientifiques de niveau recherche, publiés ou non, émanant des établissements d'enseignement et de recherche français ou étrangers, des laboratoires publics ou privés. 


\section{LE SÉNAT RÉPUBLICAIN REPRÉSENTANT DES COLLECTIVITÉS TERRITORIALES ?}

\section{Tiphaine Le Yoncourt}

Maître de conférences en histoire du droit

Faculté de droit et de science politique de l'Université de Rennes 1

Centre d'histoire du droit - IODE (UMR CNRS 6262)

Les articles adoptés par l'Assemblée nationale les 22 et 23 février 1875 fixant la composition de la seconde chambre ont sans aucun doute contribué pour une grande part à la fondation de la $\mathrm{III}^{\mathrm{e}}$ République et, au-delà, à l'implantation durable du régime républicain en France $^{1}$. Ce texte est peut-être l'autre amendement Wallon puisque c'est encore une fois au député du Nord que l'on doit la proposition qui rassemble une majorité au sein de l'Assemblée et introduit surtout une disposition résolument républicaine dans la loi constitutionnelle sur le Sénat votée deux jours plus tard. En disposant que «le Sénat se compose de trois cents membres : deux cent vingt-cinq élus par les départements et les colonies, et soixante-quinze élus par l'Assemblée nationale », l'article $1^{\mathrm{er}} \mathrm{du}$ contre-projet d'Henri Wallon fait de la seconde chambre une assemblée élue et - en ce sens - républicaine par opposition aux propositions de sénats peuplés surtout de membres de droit ou nommés que défendent alors les monarchistes ${ }^{2}$. Les articles suivants placent les conseillers municipaux au cœur de la composition du Sénat et, ce faisant, garantissent l'avènement à court terme d'une seconde chambre républicaine, car c'est dans les communes que les républicains sont en train de gagner la bataille du régime. Le «Sénat Wallon » a finalement été le fondateur et le défenseur de la République que ses promoteurs espéraient ${ }^{3}$. La chambre haute va à plusieurs reprises leur donner raison au cours de la III ${ }^{\mathrm{e}}$ République ${ }^{4}$.

1. Journal officiel, Assemblée nationale, séance du 22 février 1875, p. 1372-1386.

2. Cf. Karen Fiorentino, La Seconde Chambre en France dans l'histoire des institutions et des idées politiques (1789-1940), Paris, Dalloz, 2008, p. 405-409.

3. «Comme force de résistance, comme instrument de contrôle du pouvoir, comme frein modérateur de ce même pouvoir, c'est le Sénat qui sera votre refuge et votre ancre de salut » (Léon Gambetta, discours du 18 janvier 1876 à Aix, in Discours et plaidoyers politiques de M. Gambetta, Paris, Charpentier, 1882, t. 5, p. 46). 4. Cf. Karen Fiorentino, La Seconde Chambre..., op. cit., p. 447-512; sur la période de l'entre-deuxguerres, cf. Gisèle Bertein, Le Sénat sous la III République. 1920-1940, Paris, CNRS Éditions, 2014, p. 193-440. 
Le lien consubstantiel entre élus locaux et sénateurs établi en 1875 bénéficie donc d'une aura républicaine et constitue surtout l'identité même du Sénat, puisque c'est ce mode de désignation original qui le distingue de la chambre basse. C'est là que loge la «spécificité sénatoriale $»^{5}$ au nom de laquelle nombre de sénateurs souhaitaient récemment que l'interdiction du cumul d'un mandat exécutif local et d'un mandat parlementaire prévue par la loi du 14 février 2014 ne s'applique pas à eux ${ }^{6}$. Reste que, parmi les reproches nombreux adressés au Sénat républicain, une bonne partie renvoient précisément à son rapport aux collectivités territoriales. Il est notamment accusé de ne représenter que les collectivités territoriales et de n'assurer qu'une représentation partielle de la réalité de la nation, dont la complexité ne peut se réduire à ses élus locaux ${ }^{7}$. Mais il est également suspecté d'être une chambre aux réflexes corporatistes faisant primer les intérêts des collectivités et des élus locaux sur l'intérêt général ou les droits et libertés individuelles ${ }^{8}$. Il lui est aussi beaucoup reproché de mal représenter ces collectivités territoriales parce que désigné selon un mode de scrutin déconnecté de la démographie des territoires ${ }^{9}$ et de la réalité de la vie locale du $\mathrm{XXI}^{\mathrm{e}}$ siècle ${ }^{10}$, où les régions et intercommunalités tiennent désormais une place plus

5. François Robbe, «Le sénat défenseur des collectivités locales : histoire d'une appropriation », Pouvoirs locaux, $\mathrm{n}^{\circ}$ 67, décembre 2015 , p. 54-64.

6. Observations de sénateurs au Conseil constitutionnel à propos de la loi organique interdisant le cumul de fonctions exécutives locales avec le mandat de député ou de sénateur. Cf. décision n²014-689 DC du 13 février 2014, Conseil-constitutionnel.fr.

7. C'est, en dernier lieu, le reproche adressé au Sénat par le groupe de travail sur l'avenir des institutions qui propose sa fusion avec le Conseil économique, social et environnemental dans son rapport d'octobre 2015 Refaire la démocratie, en soulignant que "l'importance de la société civile et des corps intermédiaires est aujourd'hui trop essentielle pour que les représentants désignés par les organisations syndicales, patronales, agricoles, mutualistes, les fédérations d'associations familiales, étudiantes et environnementales demeurent confinés dans une institution dont la voix ne porte pas » (p. 103).

8. C'est la crainte qu'exprimait François Robbe en 2003 en prévenant les sénateurs par rapport au «spectre d'un sénat corporatiste» («Le Sénat à l'heure des demi-réformes», Revue française de droit constitutionnel, $\mathrm{n}^{\circ} 56,2003$, p. 725-757).

9. Jean Grangé. «Le poids des campagnes au Sénat. Effets et problèmes de la surreprésentation rurale », Économie rurale, $\mathrm{n}^{\circ} 237,1997$, p. 22-26.

10. Robert Savy, «Plaidoyer pour un Sénat du XXI ${ }^{e}$ siècle », Pouvoirs locaux, $\mathrm{n}^{\circ}$ 67, décembre 2015, p. 100-103. 
importante que les départements et les communes ${ }^{11}$. Le caractère apparemment contradictoire de ces attaques révèle toute l'ambiguïté du statut de représentant des collectivités locales du Sénat et la difficulté persistante à le penser et à le traduire concrètement dans la République française.

La «représentation des collectivités locales » par le Sénat confronte en effet la France aux contradictions et difficultés d'adaptation d'une de ses traditions révolutionnaires les plus marquantes: le principe de l'unité politique de la nation dont la volonté, toujours exclusivement générale, doit se former par l'addition des volontés individuelles des citoyens $^{12}$. Or, par son existence même, le bicamérisme instauré en 1875 dit que le suffrage universel individuel direct qui désigne les députés n'est pas le seul mode possible de représentation de la nation en République ${ }^{13}$. Plus encore, il n'assure pas une représentation complète de la nation puisqu'une seconde chambre désignée différemment est nécessaire pour former le Parlement. Nourris des conceptions organiques de la société développées au $\mathrm{XIX}^{\mathrm{e}}$ siècle, les députés de l'Assemblée nationale exprimaient cette idée ainsi : «Faire représenter la société, au sommet de l'État, par deux grandes assemblées, représentant l'une la nation à l'état de molécules, c'est la Chambre des députés, l'autre la nation à l'état organique ou dans ses organes vivants, c'est le Sénat ${ }^{14}$. $»$ En 1875, s'appuyer sur les élus locaux a permis de républicaniser le Sénat mais, dès la fin du $\mathrm{XIX}^{\mathrm{e}}$ siècle, les enjeux politiques ont changé et deux questions se posent: les collectivités locales sont-elles les seuls organes vivants de la nation qui doivent être représentés au Sénat ? La question est récurrente et laisse l'impression que la désignation des sénateurs par des élus locaux perdure en partie par défaut, figée par une tradition révolutionnaire refusant d'accorder la capacité de représenter la nation

11. Le rapport d'octobre 2015 du groupe de travail sur l'avenir des institutions à nouveau : « Pour que les membres élus de ce Sénat soient réellement représentatifs de la diversité des élus et des collectivités territoriales, il pourrait être ainsi proposé de régionaliser leur échelon d'élection » (Refaire la démocratie, op. cit., p. 104).

12. Pierre Rosanvallon, Le Modèle politique français. La société civile contre le jacobinisme de 1789 à nos jours, Paris, Seuil, 2004.

13. Cf. François Robbe, La Représentation des collectivités territoriales par le Sénat. Étude sur l'article 24, alinéa 3, de la Constitution française du 4 octobre 1958, Paris, LGDJ, 2001.

14. Pierre Pradié, Proposition de loi sur le mode de nomination d'une seconde chambre et sur l'organisation et les attributions des pouvoirs publics, 15 novembre 1873 (Journal officiel du 28 novembre 1873, annexe $\mathrm{n}^{\circ}$ 2010, p. 7263). 
aux «corps intermédiaires » qui, entre l'individu et l'État, structurent la société. Plus fondamentalement encore, la présence des élus locaux dans le collège électoral sénatorial confère-t-elle une nature politique aux collectivités locales et, partant, les sénateurs sont-ils représentants au sein du Parlement de corps politiques distincts de l'État central? L'ambiguïté persistante du Sénat provient aussi de la difficulté pour la République française à reconnaître les collectivités locales autrement que comme des subdivisions administratives d'un tout.

\section{La désignation des sénateurs par les élus locaux : tradition républicaine ou choix par défaut?}

Une fois les républicains établis au pouvoir dans la décennie 1880, réclamer l'organisation d'une forme de représentation professionnelle au Sénat n'est plus synonyme de retour à l'Ancien Régime. S'ouvre alors une discussion toujours en cours sur la nature juridique et politique des « organes vivants », des « forces vives» de la nation, sur les moyens d'organiser une «démocratie pluraliste », « sociale » ou «participative » par l'intermédiaire du Sénat.

L'un des premiers à plaider dans ce sens est Jean Jaurès en $1889^{15}$. Ce faisant, il témoigne, sinon d'une conversion des socialistes au bicamérisme, du moins de l'évolution d'une partie d'entre eux à l'heure où le Sénat se fait défenseur de la République en pleine crise boulangiste $^{16}$. Une seconde chambre pourquoi pas, mais une chambre du travail, élue dans le cadre départemental autour de trois catégories: l'industrie, le commerce et l'agriculture. Elle serait ainsi «l'image exacte de la nation tout entière », dit Jaurès ${ }^{17}$ : «Admettez un moment que cette chambre du travail se substitue au Sénat actuel oligarchique et sans racines profondes dans le pays, et voyez quelle solide base vous donnez à la République. » Quelques années plus tard, Léon Duguit suggère lui aussi de transformer le

15. Cf. Alain Chatriot, «Jaurès face au Sénat. La chambre haute : problème ou solution pour les socialistes et les républicains », Cahiers Jaurès, n 174, 2004, p. 39-52.

16. Cf. Karen Fiorentino, La Seconde Chambre..., op. cit., p. 499-500.

17. Jean Jaurés, «La Chambre du travail », La Dépêche, 13 janvier 1889 ; disponible sur « Articles sur la réforme du parlementarisme français (1887-1896) », JusPoliticum.com. 
mode de composition du Sénat, mais sa logique est différente puisqu'il ne propose pas d'organiser des élections catégorielles mais d'établir une chambre des groupes sociaux ${ }^{18}$. Ce renouvellement de la représentation de la nation lui semble possible dans la mesure où, estime-t-il, l'individualisme révolutionnaire s'écroule - ce dont il se félicite - et que depuis vingt ans se développe ce qu'il appelle «l'associationnisme », preuve qu' « une société ne se compose pas seulement d'individus isolés placés seuls en face de l'État, mais encore de groupements, d'unions organiques ayant leur vie propre distincte de la vie intellectuelle, et notamment de groupes ouvriers, agricoles, scientifiques, administratifs ${ }^{19}$. La République elle-même en a reconnu et organisé l'existence en légalisant les syndicats en 1884. Pour Duguit, il est désormais temps de fonder la seconde chambre sur ces organisations. En 1911, il réclame de façon plus explicite une seconde chambre syndicale, arguant de ce que, «les éléments qui occupent la place prééminente dans le système social moderne, ce sont les groupes corporatifs et syndicaux, et qu'on n'aura rien fait pour l'organisation de la représentation politique tant qu'on n'aura pas assuré au Parlement la représentation de ces groupes corporatifs ou syndicaux ${ }^{20} \gg$.

Les projets de réorganisation de la composition du Sénat substituant ou ajoutant aux élus locaux des représentants des organisations collectives professionnelles existent donc dès la fin du XIX ${ }^{\mathrm{e}}$, mais n'aboutissent pas $^{21}$. D'abord parce qu'une réforme du Sénat suppose que le Sénat lui-même y consente. Ensuite et plus fondamentalement parce qu'une telle redéfinition suppose de rompre avec la tradition révolutionnaire qui interdit d'intégrer la représentation d'intérêts catégoriels dans la représentation nationale. La force de cette tradition est particulièrement sensible dans l'entre-deux-guerres. La représentation professionnelle est plus que jamais d'actualité, relayée désormais par certains hauts fonctionnaires tel le conseiller d'État Henri Chardon qui voit dans l'instauration d'un conseil supérieur du travail national

18. Claude Didry, «De l'État aux groupes professionnels. Les itinéraires croisés de L. Duguit et É. Durkheim au tournant du siècle (1880-1900)», Genèses, n 2, 1990, p. 5-27.

19. Léon Duguit, «L'élection des sénateurs », Revue politique et parlementaire, $\mathrm{n}^{\circ}$ 11, 1895, p. 463.

20. Léon Duguit, «La représentation syndicale au Parlement », Revue politique et parlementaire, $\mathrm{n}^{\circ} 3$, 1911, p. 28-45.

21. Sur les projets de Sénat corporatiste dans les dernières années du siècle, cf. François Robbe, $L a$ Représentation des collectivités territoriales..., op. cit., p. 109-127. 
l'un des éléments de la réforme de l'État alors réclamée par tous ${ }^{22}$. Mais pour Chardon comme pour la majorité de ses contemporains ${ }^{23}$, cette représentation professionnelle n'est acceptable que dotée de compétences consultatives et en dehors du Parlement. C'est le cas du Conseil national économique instauré en 1925, la plus célèbre illustration de cette tendance ${ }^{24}$.

L'instrumentalisation du corporatisme par le gouvernement de l'État français ${ }^{25}$ condamne le mot et change évidemment le contexte politique dans lequel la représentation professionnelle est débattue après 1945. Mais, sous le terme de "démocratie économique et sociale» consacré par le Conseil national de la Résistance, elle compte parmi les préoccupations des constituants de 1946. La première constituante fait le choix du monocamérisme et s'inscrit logiquement dans la continuité de la III $^{\mathrm{e}}$ République en la matière ; elle propose de constitutionnaliser un conseil économique consultatif. Cependant, la minorité favorable au bicamérisme plaide alors pour une seconde chambre pluraliste. « Nous voudrions qu'un tiers en soit élu par les collectivités locales, telle que les organise le titre VIII du projet de Constitution ; qu'un autre tiers soit élu par les grandes centrales syndicales et les organisations économiques; que le dernier tiers enfin soit élu par les associations familiales qu'a organisées, en mars 1945, le gouvernement de la Libération », explique Paul CosteFloret au nom du Mouvement républicain populaire (MRP) ${ }^{26}$. Mais, en juin 1946 à Bayeux, de Gaulle semble être le seul à continuer de défendre l'idée d'une seconde chambre législative qui compterait aussi «des représentants des organisations économiques, familiales, intellectuelles, pour que se fasse entendre, au-dedans même de l'État, la voix des grandes activités du pays ${ }^{27}$. Après l'échec du référendum du 5 mai, le MRP a renoncé à la composition pluraliste de la seconde chambre, car le bicamérisme ne semble acceptable par la nouvelle constituante que dans une forme classique, c'est-à-dire appuyée sur les élus locaux.

22. Henri Chardon, L'Organisation de la République pour la paix, Paris, PUF, 1926, p. 74-77.

23. Alain Chatriot, «Les apories de la représentation de la société civile. Débats et expériences autour des compositions successives des assemblées consultatives en France au $\mathrm{XX}^{\mathrm{e}}$ siècle », Revue française de droit constitutionnel, $\mathrm{n}^{\circ} 71,2007$, p. 535-555.

24. Alain Chatriot, La Démocratie sociale à la française. L'expérience du Conseil national économique (1924-1940), Paris, La Découverte, 2002.

25. Jean-Pierre Le Crom, Syndicats, nous voilà ! Vichy et le corporatisme, Paris, L’Atelier, 1995.

26. Journal officiel, Assemblée nationale constituante, séance du 9 avril 1946, p. 1640.

27 Discours de Bayeux du 16 juin 1946, in Mémoires d'espoir, Paris, Plon, 1999, p. 313 
Le projet voté par l'Assemblée puis par les Français par référendum dispose donc que le Conseil de la République, aux compétences politiques pour l'instant très restreintes, est élu par les collectivités communales et départementales, au suffrage universel indirect. La représentation professionnelle reste cantonnée dans un conseil économique bien distinct du Parlement, qui seul représente la nation.

En 1958, le processus se renouvelle de façon étonnamment similaire. Les éléments compilés dans les archives des rédacteurs de la Constitution montrent que, jusqu'à la mi-juin 1958, la seconde chambre devait se composer de trois sections : celle des collectivités locales de métropole, celle des territoires d'outre-mer et celle des forces économiques, sociales et culturelles $^{28}$. Elles montrent également que cette organisation de l'autre représentation de la nation est le souhait du général de Gaulle ${ }^{29}$ mais que la section dite «économique » inquiète plusieurs membres du groupe de travail, aussi bien Michel Debré, qui « craint la politisation des forces économiques », que Guy Mollet, qui estime «que son recrutement par les organisations syndicales serait contraire au principe selon lequel le pouvoir législatif dérive uniquement du suffrage universel $»^{30}$. C'est cette conception classique de la représentation nationale qui s'impose in fine. Le Conseil économique et social est maintenu dans sa forme antérieure et la section économique du Sénat n'apparaît plus dans les projets à partir de la fin juin 1958. Il semble que, contrairement à ce qu'affirmait Duguit en 1911, la consécration légale des organisations collectives au tournant des $\mathrm{XIX}^{\mathrm{e}}$ et $\mathrm{XX}^{\mathrm{e}}$ siècle n'était motivée que par un «impératif de gouvernabilité », pour reprendre la formule de Pierre Rosanvallon ${ }^{31}$. L'État républicain a donné un cadre juridique aux syndicats, associations, fédérations et groupements de tous types sur lesquels il s'appuie et avec lesquelles il gouverne. Mais, ce faisant, il ne les reconnaît pas en tant qu'organes politiques participant à la représentation de la nation. Dans la tradition du droit public français et de ses principes révolutionnaires, l'expression d'intérêts

28. Didier Maus (dir.), Documents pour servir à l'histoire de l'élaboration de la Constitution du 4 octobre 1958, Paris, La Documentation française, 2002, t. 1, p. 246.

29. Cf. François Robbe, La Représentation des collectivités territoriales..., op. cit., p. 132-148.

30. «Compte rendu de la réunion constitutionnelle du 13 juin $1958 »$, in Didier Maus (dir.), Documents pour servir à l'histoire..., op. cit., t. 1, p. 247.

31 Pierre Rosanvallon, Le Modèle politique français, op. cit., p. 129 
particuliers, catégoriels, ne saurait participer à la production de la volonté générale. Contre Léon Duguit, ce sont les idées d'Adhémar Esmein qui l'emportent encore ${ }^{32}$.

Le projet avorté de 1969 ne s'écartait que peu de cette ligne ${ }^{33}$. Plus qu'une réforme de la composition de la seconde chambre législative, il s'agissait d'une remise en cause du bicaméralisme à saisir dans une atmosphère de conflit entre de Gaulle et le Sénat, et dans la continuité du renforcement de l'exécutif de $1962^{34}$. Le projet propose de fusionner Conseil économique et social et Sénat. Néanmoins, contrairement à ce que de Gaulle proposait dans le discours de Bayeux de juin 1946 ou ce qu'il défendait au printemps 1958, il ne s'agit pas seulement d'ouvrir le Parlement aux «forces vives » ${ }^{35}$ de la nation, mais également d'écarter la seconde chambre du processus législatif en mêlant représentants des collectivités locales et des organisations professionnelles et familiales dans un organe chargé de conseiller l'Assemblée nationale, seule institution politique ${ }^{36}$. Lorsqu'en 2015 le groupe de travail sur l'avenir des institutions propose la fusion du Conseil économique, social et environnemental et du Sénat, il prend d'ailleurs soin de souligner que son projet s'écarte de celui de 1969 en ce que le Sénat conserverait sa compétence législative. Mais «faire du Sénat un représentant d'objets inanimés impliquera de donner à ceux-ci une âme, avec toutes les conséquences juridiques que cela emporte », prévient Alain-Gérard Slama ${ }^{37}$. L’image exprime bien le bouleversement de principe que la réforme porte en elle, et la proposition n'est d'ailleurs

32. «Le principe de la souveraineté nationale exclut, dans le suffrage politique et en droit, ce qu'on appelle la représentation des intérêts. [...] Un pareil système ne saurait se concilier avec le principe de la souveraineté nationale, d'après lequel les citoyens électeurs, absolument égaux entre eux, ont nécessairement le même titre et la même qualité. La souveraineté nationale, c'est, en droit, la négation de tout système de classes » (Adhémar Esmein, Éléments de droit constitutionnel, Paris, Larose \& Forcel, 1896, p. 183).

33. Décret du 2 avril 1969 décidant de soumettre au référendum un projet de loi relatif à la création de régions et à la rénovation du Sénat (Journal officiel du 3 avril 1969, p. 3315).

34. Cf. Jean Mastias, Le Sénat de la Veépublique. Réforme et renouveau, Paris, Economica, 1980, p. 222310 ; Fabien Conord, «De la "République sénatoriale" à la "forfaiture". Le Sénat et la Ve République, 1959$1962 »$, Histoire-politique.fr, 2010.

35. Alain Chatriot, «À la recherche des "forces vives" », in Serge Bertein, Pierre Birnbaum, Jean-Pierre Rioux (dir.), De Gaulle et les élites, Paris, La Découverte, 2008, p. 219-237.

36. Cf. Jean Mastias, Le Sénat de la Ve République, p. 337-363.

37. «Audition de M. Bruno Latour, professeur à Science Po, sur le thème de la démocratie sociale, le 5 février 2015 », Refaire la démocratie, op. cit., p. 420. 
adoptée qu'à une courte majorité de 12 voix contre 9 et 2 abstentions. Cependant, si l'on veut filer la métaphore, l'interrogation lamartinienne surgit : collectivités locales, avez-vous donc une âme?

\section{Le Sénat républicain ou la nature ambiguë des collectivités locales}

En 1874, le premier projet de la commission constituante sur la composition du Sénat avait écarté toutes les propositions intégrant les conseillers municipaux et généraux dans le collège sénatorial ainsi que celles prévoyant la désignation de délégués sénatoriaux par les conseils municipaux ou de département. «Il lui a paru qu'il importait de ne donner aucune attribution politique à des conseils administratifs », explique le rapporteur ${ }^{38}$. La commission relaie ici le refus de la majorité de l'Assemblée nationale de reconnaître les départements et surtout les communes comme des espaces politiques. Six mois plus tard, la commission change d'avis sous la pression des républicains pour qui le collège sénatorial doit être composé d'élus, mais elle est surtout motivée par un calcul politique qui échouera ${ }^{39}$. Mais en faire des électeurs nationaux ne remet pas en cause la nature purement administrative des conseils municipaux. L'instauration de ce Sénat appuyé sur les élus locaux ne contredit évidemment pas le caractère unitaire de l'État français; les deux chambres, séparément et ensemble, représentent la nation. Si les sénateurs représentent quelque chose en plus, il ne peut s'agir que du territoire du département. Il est en effet le cadre du collège sénatorial au sein duquel voisinent les délégués des conseils municipaux, les conseillers d'arrondissement et généraux en tant qu'élus au suffrage universel et non comme délégués de leurs collectivités, et enfin les députés du département qui ne sont évidemment issus d'aucune collectivité locale ${ }^{40}$. Les Républicains ont beau soutenir que, la commune, «c'est la démocratie en personne ayant ses intérêts, ses aspirations, son patrimoine, dont la gestion est

38. Antonin Lefèvre-Pontalis, rapport fait au nom de la commission des lois constitutionnelles sur les projets de lois et les propositions concernant la création et les attributions d'un Sénat, Assemblée nationale, séance du 3 août 1874 (Journal officiel du 24 décembre 1874, annexe $n^{\circ} 2680$, p. 8554).

39. Étant donné le nombre des conseils municipaux compatibles avec le gouvernement d'ordre moral, « la commission a pensé qu'il y avait bien plus à gagner qu'à perdre à les intéresser à l'existence du Sénat », déclare Antonin Lefèvre-Pontalis (Journal officiel, Assemblée nationale, séance du 22 février 1785, p. 1373).

40. Cf. Karen Fiorentino, La Seconde Chambre..., op. cit., p. 440-442. 
confiée à un conseil municipal élu par tous les habitants, auquel, jusqu'ici, il a été interdit de s'occuper de politique ${ }^{41} »$, l'établissement d'un Sénat composé par les élus locaux n'est en aucun cas la concrétisation ni même l'annonce de la décentralisation. En formant l'essentiel de l'électorat sénatorial, les élus locaux sont consacrés comme notables de la République, mais les conseils locaux n'en retirent aucune forme d'autonomie.

Les débats autour de la composition du Conseil de la République en 1946 et au début de la IV ${ }^{\mathrm{e}}$ République démontrent que la situation des collectivités locales et leur rapport avec la seconde chambre n'ont pas changé au milieu du $\mathrm{XX}^{\mathrm{e}}$ siècle $^{42}$. Pendant l'été 1946, deux doctrines s'affrontent à ce propos: la première, soutenue pour l'essentiel par les communistes, réclame le recours à de grands électeurs désignés au suffrage universel direct. La seconde, appuyée par les radicaux, le MRP, la droite et le Rassemblement des gauches républicaines, plaide pour un collège électoral composé d'élus locaux, adaptation du système de $1884^{43}$. Il s'agirait ici d'établir un Conseil de la République qui «représente les collectivités locales, c'est-à-dire émane des conseils généraux et des conseils municipaux » car, «dans un pays comme la France, les collectivités locales jouent un rôle analogue, toutes proportions gardées, à celui que tiennent les États membres dans les États fédéraux », affirme Paul Bastid ${ }^{44}$. La formule «toutes proportions gardées » est évidemment un euphémisme. Les défenseurs d'une telle seconde chambre n'entendent pas en faire le lieu d'expression d'entités politiques autonomes au sein de la représentation nationale. Quelques jours plus tôt, le même Paul Bastid demandait d'ailleurs la suppression du titre $X$, qui dispose notamment que «les collectivités territoriales s'administrent librement par des conseils élus au suffrage universel » au nom du principe d'unité nationale et par refus de la décentralisation que la commission prétendait engager en constitutionnalisant les collectivités territoriales ${ }^{45}$.

\footnotetext{
41. Léon Gambetta, discours du 18 janvier 1876 à Aix, discours cité, p. 34.

42. Cf. François Robbe, La Représentation des collectivités territoriales..., op. cit., p. 307-315.

43. Cf. les débats au sein de la commission de la Constitution résumés par le rapporteur Maurice Boisdon (Journal officiel, Assemblée nationale constituante, séance du 27 septembre 1946, p. 4150-4152).

44. Journal officiel, Assemblée nationale constituante, séance du 11 septembre 1946, p. 3646.

45. Cf. le débat à ce sujet (Journal officiel, Assemblée nationale constituante, séance du 5 septembre 1946, p. 3559-3562).
} 
S'appuyer sur les élus locaux pour composer la seconde chambre en 1946, ce n'est donc qu'établir un mode de scrutin destiné à écarter les grands électeurs défendus par les communistes. Mais cela ne dit toujours rien des collectivités elles-mêmes et ne change pas leur rôle de gestion purement administrative de la nation. Le retour en $1948^{46}$, après une période transitoire $^{47}$, à un mode de composition traditionnel par des élus locaux renforce sans doute l'autorité du Conseil et, conjugué à un contexte politique plus favorable au bicamérisme, facilite son avènement progressif au rôle de seconde chambre législative. Ce n'est cependant pas la représentation des collectivités territoriales qui fonde sa légitimité ou définit sa mission. Ce sont les sénateurs eux-mêmes, puisqu'ils ont retrouvé ce nom, et les élus qui composent leur collège électoral qui, individuellement, confèrent de l'autorité au Conseil. À la fin de l'année 1948, Gaston Monnerville affirme d'ailleurs que l'assemblée qu'il préside se distingue de la chambre basse en ce qu'elle puise «son origine dans des collèges électoraux composés d'administrateurs expérimentés, rompus à la gestion des affaires locales, habitués à travailler dans un cadre sans doute plus restreint [...] où s'affirment, avec une force particulière, les réalités concrètes qui peuvent échapper à une vue plus générale des problèmes ${ }^{48}{ }$. Le Conseil de la République interprète d'ailleurs sa mission, certes, comme celle de représentant des collectivités locales en tant que corps, mais surtout comme celle de protecteur de la France rurale, de défenseur du monde paysan contre celui de l'industrie, et s'appuie souvent sur les débats agricoles pour affirmer son droit de regard sur les textes votés à la chambre haute ${ }^{49}$.

En 1958, les articles de la Constitution concernant la seconde chambre n'ont plus à faire le compromis entre partisans de la désignation par les grands électeurs ou par les élus locaux. Mais la rédaction de 1'article 24-3 doit être replacée dans les circonstances coloniales du moment et mise en lien avec l'ambition gaulliste d'organiser en fédération les territoires

46. Loi 48-1471 du 23 septembre 1948 relative à l'élection des conseillers de la république (Journal officiel du 24 septembre 1948, p. 9394).

47. Par souci de compromis, la loi électorale provisoire de 1946 mêle au sein du collège sénatorial conseillers généraux, députés du département et délégués désignés par canton au suffrage universel (Loi sur la composition et l'élection du conseil de la république, Journal officiel du 28 octobre 1946, p. 9176).

48. Journal officiel, Conseil de la République, séance du 25 novembre 1948, p. 3479.

49. Cf. Marc Baroli et Dominique Robert, Du Conseil de la République au Sénat (1946-1958), Paris, PUF, 2002. 
rattachés à la France ${ }^{50}$. Dans les avant-projets du printemps et de l'été 1958, le Sénat est en effet une institution complexe qui «assure la représentation des collectivités territoriales de la République et celle des territoires qui font partie de la fédération », à la fois seconde chambre législative et sénat fédéral. La représentation des territoires renvoie alors à des compétences spécifiques. Ce Sénat siège en effet en assemblée générale pour traiter des affaires communes à toutes les collectivités et «les élus des départements et des territoires d'outre-mer de la République délibèrent séparément sur les affaires qui leur sont propres ${ }^{51}{ }^{1}$. Début août 1958 , le comité consultatif décide de réserver les alinéas du futur article 24 relatifs à la composition du Sénat et à la ventilation des compétences entre ses différentes formations jusqu'à ce que l'organisation de la fédération soit établie. Cette fois-ci, la désignation des sénateurs et leurs fonctions sont donc liées à l'organisation institutionnelle des territoires de la République. La création d'une «assemblée de la communauté » est décidée le 12 août. Formée de délégués des parlements des États fédérés, elle est bien distincte du Parlement de la République ; le Sénat perd donc sa fonction fédérale et la partie de l'alinéa qui précisait que le Sénat assure la représentation de la fédération et celle qui répartissait ses compétences entre les deux sections disparaissent logiquement - «c'est la conséquence de tous les textes que nous avons adoptés sur la communauté », déclare Paul Coste-Floret ${ }^{52}$. La première phrase de l'alinéa réservé, elle, est votée en l'état. C'est donc à l'issue d'un processus complexe que «le Sénat assure la représentation des collectivités territoriales de la République ». Bien sûr, l'ambition «fédérative » du projet gaulliste ne concernait que les territoires d'outre-mer. Pour ses rédacteurs, la référence à la notion de représentation dans 1'article 24-3 ne signifie pas que le Sénat est la partie du Parlement où des entités juridiques distinctes de l'État sont représentées et où leur autonomie est défendue. Comme le souligne François Robbe ${ }^{53}$, l'abandon de l'amendement qui conférait au Sénat un pouvoir de blocage sur les législations portant atteinte aux libertés des collectivités territoriales prouve que le gouvernement fait le choix

50. Cf. Jean Mastias, Le Sénat de la Ve République, op. cit., p. 199-200.

51. Article 22 de l'avant-projet du 19 juillet 1958 mis au point après les réunions du conseil interministériel des 16, 17 et 18 juillet et que l'on retrouve dans l'avant-projet soumis au conseil de cabinet les 23 et 25 juillet ou encore dans l'avant-projet des 26-29 juillet. Cf. Didier Maus (dir.), Documents pour servir à l'histoire..., op. cit., t. 1, p. 461.

52. Comité consultatif constitutionnel du 13 août 1958, ibid., p. 454.

53. La Représentation des collectivités territoriales..., op. cit., p. 328-330 
délibéré d'écarter cette lecture. Produit d'un hasard de rédaction, scorie d'un projet institutionnel avorté, reste que la Constitution de 1958 use pour la première fois du terme de représentation pour évoquer le rapport entre collectivités territoriales et Sénat. Si l'intention des rédacteurs d'y voir la constitutionnalisation d'une tradition électorale républicaine est claire, rien n'interdit de donner ensuite une autre dimension au terme, notamment lorsque les législations de la fin du $\mathrm{XX}^{\mathrm{e}}$ et du début du $\mathrm{XXI}^{\mathrm{e}}$ siècle transforment la nature des collectivités territoriales.

Dès la fin des années 1970, la décentralisation cesse d'être une réorganisation administrative de l'État mal distinguée de la déconcentration ${ }^{54}$ pour devenir une remise en cause politique de l'appareil d'État ${ }^{55}$. Les réformes législatives, l'interprétation qu'en donne le Conseil constitutionnel et le développement du droit constitutionnel des collectivités territoriales ${ }^{56}$ sont la traduction de leur reconnaissance en tant que corps politiques. Le Sénat participe à la mutation des collectivités locales ${ }^{57}$ mais, en retour, elle semble peu transformer le Sénat. La réforme de 2003 reste limitée ${ }^{58}$, et l'étude des discours sénatoriaux pendant la première décennie du XXI ${ }^{\mathrm{e}}$ siècle le montre : ce que les sénateurs représentent reste flou et la référence aux collectivités locales mêle toujours confusément les territoires, la ruralité, les élus locaux et les entités institutionnelles que sont communes, départements et régions ${ }^{59}$. Il serait toutefois trop simple d'imputer aux sénateurs l'impression d'anachronisme que dégage parfois l'institution dans la France décentralisée. «Si l'organisation jacobine première a fortement été corrigée, la culture politique de la généralité est restée dans les têtes avec toutes

54. Comme en témoignent les propos de De Gaulle lui-même pour qui «il faut maintenant que, par la voie de la décentralisation, le préfet de région dispose des moyens administratifs adéquats » (conférence de presse du 9 septembre 1968, in Mémoires d'espoir, Paris, Plon, 1999, p. 1105).

55. Cf. Philippe Bezes, Réinventer l'État. Les réformes de l'administration française, 1962-2008, Paris, PUF, 2009, p. 175-180.

56. Cf. Michel Verpeaux, « Le droit constitutionnel des collectivités territoriales, avant-propos » (dossier), Cahiers du Conseil constitutionnel, $\mathrm{n}^{\circ} 12$, mai 2002.

57. Sur le rôle du Sénat dans la décentralisation, cf. Alain Delcamp, Le Sénat et la Décentralisation, Paris, Economica, 1991 ; plus récemment, Sophie Lamouroux, «Les relations entre le Sénat et la décentralisation », DroitConstitutionnel.org.

58. Cf. François Robbe, « Le Sénat à l'heure des demi-réformes », art. cité.

59. Cf. Benjamin Morel, «Le représenté introuvable. Tentatives et paradoxe d'une représentation sénatoriale en France », Congres-AFSP.fr. 
les conséquences en termes de conception de la souveraineté ou de l'intérêt général ${ }^{60}$. » Le Sénat, chargé de représenter la nation souveraine autrement que par le suffrage universel individuel direct sans jamais prétendre exprimer autre chose que la volonté générale, est ainsi prisonnier d'une tradition plus ancienne que lui et qui, dans l'état actuel du droit public français, lui interdit d'être tout à fait le représentant des collectivités territoriales autant que telles et l'empêche d'intégrer la représentation d'autres formes de corps politiques.

\section{Résumé}

Les élus locaux forment le corps électoral sénatorial depuis la IIIe République. C'est ce qui fonde le Sénat républicain et ce qui lui vaut son statut de « représentant des collectivités territoriales ». L'histoire de la seconde chambre depuis 1875 révèle cependant toute l'ambiguïté de la formule. Les principes hérités de la révolution interdisent en effet toute représentation d'intérêts particuliers ou catégoriels au Parlement. La désignation des sénateurs par les élus locaux a donc été maintenue en partie par défaut, faute d'alternative légitime, mais sans pour autant ériger les collectivités territoriales «représentées » au Sénat en entités politiques autonomes au sein de la Nation.

60. Pierre Rosanvallon, Le Modèle politique français, op. cit., p. 432. 